\title{
Damage spreading in the 'sandpile' model of SOC
}

\author{
Ajanta Bhowal* \\ Saha Institute of Nuclear Physics \\ 1/AF Bidhannagar, Calcutta-700064, India
}

\begin{abstract}
We have studied the damage spreading (defined in the text) in the 'sandpile' model of self organised criticality. We have studied the variations of the critical time (defined in the text) and the total number of sites damaged at critical time as a function of system size. Both shows the power law variation.
\end{abstract}

Keywords: Sandpile model of SOC, Damage spreading

PACS Numbers: $05.50+q$

*Present address:Department of Physics, Lady Brabourne College, P-1/2 Suhrawardy Avenue, Calcutta-700017, India

E-mail:ajanta.bhowal@gmail.com 


\section{Introduction}

The damage spreading [1-3] studies the dynamic behaviour of cooperative systems. The main idea of this problem is to study how a small perturbation, called a damage, in a cooperative system changes with the evolution of time. This is studied by observing the time evolution of the two copies of the system with slightly different initial configuration under the same dynamics and measuring the damage by counting the number of elements which are different in the two copies of the system. The damage spreading has been studied exhaustively, in the Kauffman model [1] and the spin systems $[2,3]$.

In this paper, we have studied, by computer simulation, how damage spreads, in the 'sandpile' model, over the whole lattice during the course of time evolution. 'sandpile' model [4] is a lattice automata model which describes the appearance of long range spatio-temporal correlations observed in extended, dissipative dynamical systems in nature. The essential feature of this model is the occurence of fractal structure in space and ' $1 / f$ ' noise in time, which is so called self-organised criticality (SOC) [4]. Substantial developments have been made on the study of 'sandpile' model. But all these studies have been made in the steady (SOC) state, reached by the system.

\section{The model and simulation}

The lattice automata 'sandpile' model [4] of SOC evolves to a stationary state in a self-organised (having no tunable parameter) way. This state has no scale of length and time, hence is called critical. Altogether the state is called self-organised critical state. The description of the lattice automata model is as follows: At each site of this lattice, a variable (automaton) $z(i, j)$ is associated which can take positive integer values. Starting from the initial condition (at every site $z(i, j)=0$ ), the value of $z(i, j)$ is increased (so called addition of one 'sand' particle) at a randomly chosen site $(i, j)$ of the lattice in steps of unity as,

$$
z(i, j)=z(i, j)+1 .
$$

When the value of $z$ at any site reaches a maximum $z_{m}$, its value decreases by four units (i.e., it topples) and each of the four nearest neighbours gets one unit of $z$ (maintaining local conservation) as follows:

$$
\begin{aligned}
z(i, j) & =z(i, j)-4 \\
z(i \pm 1, j \pm 1) & =z(i \pm 1, j \pm 1)+1
\end{aligned}
$$

for $z(i, j) \geq z_{m}$. At the boundary sites $z=0$ (dissipative; open boundary condition).

In this simulation, a square lattice of size $L \times L$ has been considered. The value of $z_{m}=4$ here. It has been observed that, as the time goes on the average value 
$(\bar{z})$ of $z(i, j)$, over the space, increases and ultimately reaches a steady value $\left(\bar{z}_{c}\right)$ characterising the SOC state.

We study here, by computer simulation, how a small perturbation spreads in time in the 'sandplie' model. We have considered a square lattice and allowed it to evolve under the dynamics until the average value $(\bar{z})$ of $z(i, j)$ reaches a steady value $\left(\bar{z}_{c}\right)$. We also have considered a 2nd lattice, which is a replica of the 1st lattice. After reaching the steady (SOC) state (characterised by the steady value of $\bar{z}$ ) we have perturbed suddenly one of the system (say the 1st lattice) by adding unity to the automaton value at the central site of the lattice, i.e, $z_{1}(l / 2, l / 2)=z_{1}(l / 2, l / 2)+1$. Then we allowed the two lattices to evolve in time by the specified dynamics in the same way (i.e, by using the same sequence of random numbers). It will be observed that both lattices (perturbed and unperturbed) give the same macroscopic behavior (the same $\bar{z}_{c}$ and same scale invariant (power law) distribution of the avalanches size). But the microscopic details (the $z(i, j)$ at any site $i, j$ at any time) of the two lattices are different. The differences in microscopic details of the two lattice are described here in terms of the "damaged sites", i.e., the sites of the perturbed lattice which are different from the unperturbed one. The damaged lattice is characterised by a variable (say $d(i, j)$ ), which is zero if the two lattices have the same $z$ (for any site $i, j$ ) value and 1 otherwise. More precisely, $d(i, j)=0$ if $z_{1}(i, j)=z_{2}(i, j)$ and $d(i, j)=1$ otherwise. The non-zero sites of the damaged lattice (i.e., $d(i, j)=1$ ) indicates the damage in this model.

\section{Results}

It has been observed that, with the evolution of time, the damage (cloud formed by the sites having $d(i, j)=1$ ) spreads and touches any one of the boundary line of the lattice at time $\tau$ (starting from the initial time when the central site of lattice 1 was perturbed by adding unity). Here we study the spreading of damage by measuring the following quantities:

(1) Minimum time $(\tau)$ taken by the damage to touch any one of the boundaries (upper or lower) of the lattice.

(2) The number $\left(M_{\tau}\right)$ of damaged sites at $\tau$-th instant.

(3) The total number $\left(M_{T o t}\right)$ of sites damaged during the time $\tau$, starting from the initial time when the perturbation was added.

Fig. 1 shows the variation of critical time $(\tau)$ with the sytem size $L$ in the log scale indicating the power law variation, following, $\tau \sim L^{a}$ with $a=1.97$. Fig.2 shows the variations of $M_{\tau}$, the number of sites damaged at critical time $(\tau)$ and $M_{t o t}$, the total number of sites damaged up to critical time with the syatem size $L$ in the log scale, indicating the power law variations, $M_{\tau} \sim L^{b}$ with $b=1.47$. and $M_{\text {tot }} \sim L^{c}$ with $c=1.66$. Thus we see that $\tau, M_{\tau}$ and $M_{\text {tot }}$, all these quantities follow a power law variation with the system size. All these data have been obtained by sampling over 
100 different seeds of random number generator for $L=30,40, \cdots 150$ and over 10 samples for $L=200,300$.

\section{Summary}

In the case of damage spreading in Ising model the spreading of damage is controlled by tuning the temperature. But due to the absence of any tunable parameter in the 'sandpile' model it has been observed that in this case there is number spreading transition contrast to the other cases of damage spreading (like Ising system etc). There is a power law variation of critical time $(\tau)$ and $M_{\tau}, M_{t o t}$ with length as in the other cases (though the exponents are different).

\section{References}

[1] A. Kauffman, J. Theor. Biol. 22, 437 (1969); See also B. Derrida and Y. Pomeau, Europhys. Lett. 1, 45 (1986); B. Derrida and G. Weisbuch, J. Phys. (Paris) 47, 1297 (1986); B. Derrida and D. Stauffer, Europhys. Lett. 2, 739 (1986).

[2] N. Jan and L. de Arcangelis, in Annual Reviews of Computational Physics, Vol 1., Ed. D. Stauffer, World-Scientific, Singapore, (1994) 1.

[3] P. Grassberger, J. Stat. Phys. 79, 13 (1995).

[4] P. Bak, C. Tang and K. Wiesenfeld, Phys. Rev. Lett. 59 (1987) 381; Phys. Rev. A. 38 (1988) 364. 


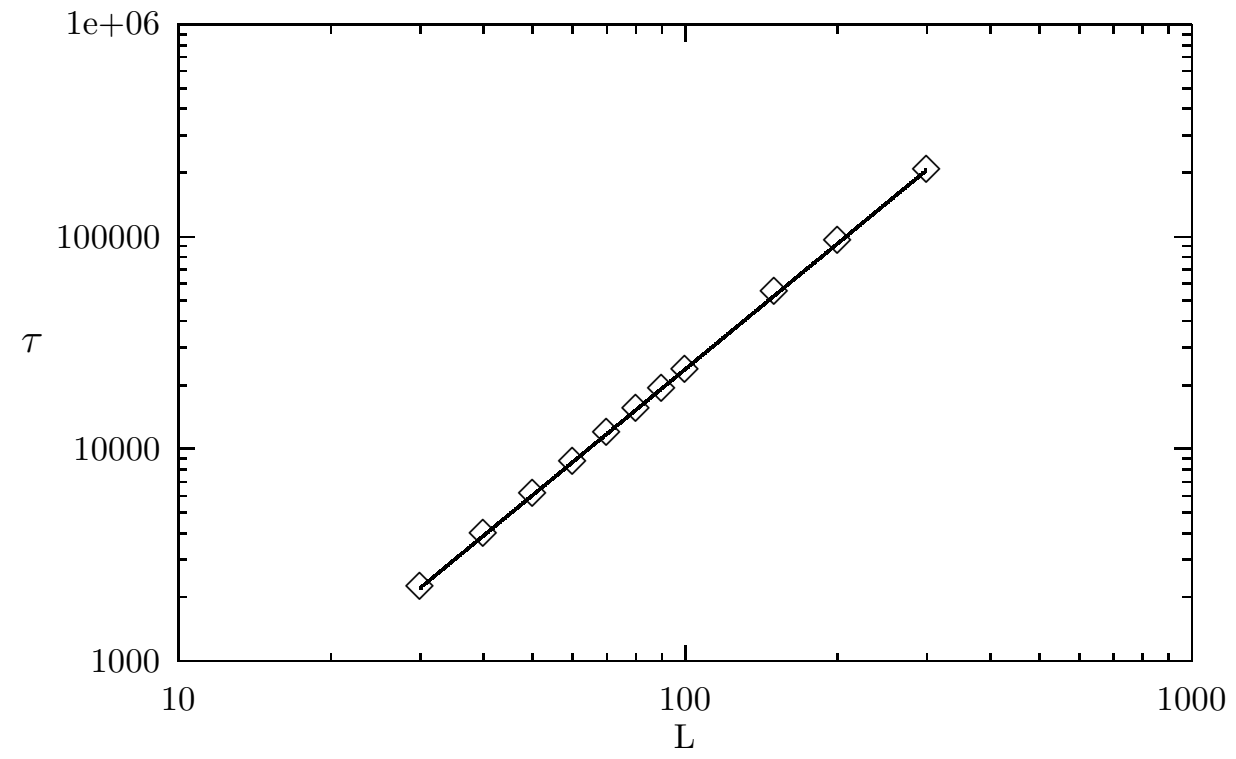

Fig. 1. Variation of critical time $(\tau)$ with system size $L$.

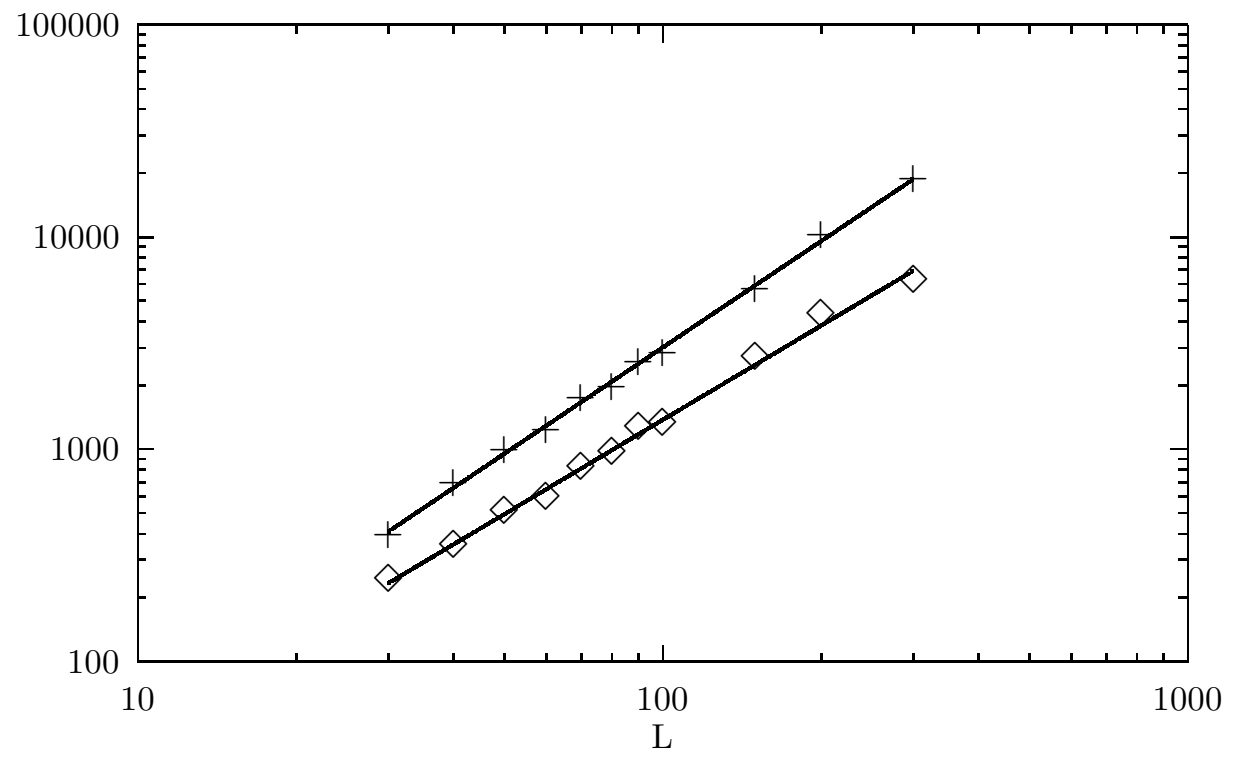

Fig. 2. Variations of $M_{\tau}(\diamond)$ and $M_{t o t}(+)$ with system size $L$. 